\title{
DE LA GUERRA DEL PELOPONESO A LA PAZ DEL REY (II): LOS ELEMENTOS DE LA CIUDADANÍA ATENIENSE*
}

\author{
Domingo Plácido y César Fornis \\ Universidad Complutense de Madrid y Universidad de Sevilla \\ placido@ghis.ucm.es y cfornis@us.es

\begin{abstract}
FROM THE PELOPONNESIAN WAR TO THE KING'S PEACE (II): ELEMENTS OF ATHENIAN CITIZENSHIP
\end{abstract}

\begin{abstract}
En el presente artículo se examinan los problemas y tensiones en torno a la definición de la ciudadanía en la Atenas de finales del siglo $\mathrm{V}$ y principios del siglo IV a. C., problemas que se relacionan con la evolución de las formas de dependencia tras la guerra del Peloponeso. Los elementos de la ciudadanía, junto a los protagonismos personales y los factores económicos públicos y privados, analizados en otros trabajos, ofrecen un panorama complejo, pero coherente, de la sociedad ateniense, en el que todos estos elementos interactúan y se explican entre sí.

Palabras clave: ciudadanía; derechos; participación; exclusión; dependencia; oligarquía; democracia.
\end{abstract}

The article discusses the problems and tensions surrounding the definition of Athenian citizenship at the end of fifth century and early fourth century $\mathrm{BC}$, which are related to the evolution in forms of dependence after the Peloponnesian War. Citizenship rights, alongside personal leadership questions and public and private economic factors, analyzed in other works, reveal a complex but consistent picture of the Athenian society, in which all of these elements interact and explain each other.

Keywords: citizenship; rights; participation; exclusion; dependence; oligarchy; democracy.

El momento de protagonismo de los individuos analizados en una primera parte de este estudio ${ }^{1}$ parece coincidir con un proceso de reducción de la población de Atenas con respecto al siglo $\mathrm{V}$ como consecuencia de la guerra del Peloponeso. Según cálculos más o menos sólidos, se piensa que en los inicios del siglo IV había entre 20.000 y 30.000 ciudadanos, mientras que en el inicio de la citada contienda se calculan unos 60.000, con lo que se habría producido la pérdida de más de la mitad de la población, entre epidemia,

* Este artículo se ha elaborado en el marco del Proyecto de Investigación HUM200761213/HIST, del Ministerio de Educación y Ciencia.

${ }^{1}$ C. Fornis y D. Plácido, «De la guerra del Peloponeso a la paz del Rey (I): prosopografía política ateniense», RSA 38, 2008, pp. 37-79. 
batallas y represión oligárquica. Ello provocó también la disminución de los nacimientos en la primera mitad del siglo $\mathrm{IV}^{2}$. Desde los primeros años, ciertos síntomas se interpretan como reacción ante dichas circunstancias, como las alusiones de Andócides I 149 (Sobre los misterios) a una propuesta no realizada de naturalización de extranjeros como compensación, en 399, en la que se pretendía hacer ciudadanos a tesalios y andrios por penuria de hombres $\left(\delta \imath^{\prime} \alpha \dot{\alpha} \pi o \rho i ́ \alpha v \dot{\alpha} v \delta \rho \tilde{\omega} v\right)$. Sí parece claro que se produjo en algún momento la suspensión de las limitaciones contenidas en la ley de la época de Pericles (451/450), referente al $\mu \eta \tau \rho o ́ \xi \varepsilon v o \zeta$, el hijo de madre extranjera, pues de Iseo VI 47 (Sobre la herencia de Filoctemón) y VIII 19 y 43 (Sobre la herencia de Cirón) se deduce que antes del arcontado de Euclides eran ciudadanos los hijos de madre no ciudadana ${ }^{3}$. Posteriormente fue reactivada, sin revisión de los nacidos antes ( $F G H 77 \mathrm{~F}$ 2), en el arcontado de Euclides (403/402), por una propuesta de ley de Aristofonte, según Ateneo (577B), lo que parece indicar que se imponen los criterios restrictivos.

Como se ve, los posibles problemas derivados de la escasez de población se interfieren con los que se relacionan con la atención a los ciudadanos tal como se practicaba en los momentos de apogeo del imperio. La carencia del pópos dificulta la redistribución sobre bases públicas que se había instalado como sistema desde tiempos de Pericles. Teozótides, según Lisias fr. 6 (Contra Teozótides), propuso una ley, en el momento inmediato a la restauración, con el intento de apartar a los bastardos de las ayudas que la ciudad concedía a los huérfanos de los ciudadanos caídos combatiendo a la oligarquía; Teozótides sometió asimismo a la Asamblea el decreto que reducía el pago de una dracma a cuatro óbolos a los caballeros, presumiblemente también tras el derrocamiento de los Treinta y por haberse significado con éstos (porque al mismo tiempo se incrementa de dos a ocho óbolos el salario

${ }^{2}$ Un estado de la cuestión en J. Oulhen, «La société athénienne», en P. Brulé y R. Descat (eds.), Le monde grec aux temps classiques. 2 Le IV siècle, París, 2004, pp. 258-260, y P. Brulé, La Grèce d'à côté. Réel et imaginaire en miroir en Grèce antique, Rennes, 2007, pp. 57-65, quien, contra la creencia generalizada de que la guerra mata, pero poco, calcula en unas 38.000 las bajas provocadas por la guerra del Peloponeso entre la población masculina ciudadana en edad adulta, más unas 18.000 entre los menores de 18 años (lo que da un total de 56.000), de modo que concluye que «la guerre athénienne a tué, et beaucoup!».

${ }^{3}$ S. Ferruci, comentario a Iseo, La successione de Kiron, Pisa, 2003, ad loc. Cf. también D. XLIII 51 (Contra Macártato), LVII 30 (Contra Eubúlides); Sch.Aeschin. I 39 (Contra Timarco); Ath. 577B. 
de los i $\pi \pi 0 \tau o \xi o ́ \tau \alpha \iota$ o arqueros a caballo). En 1970 se halló en el ágora de Atenas una estela con el decreto de los huérfanos de ciudadanos - excluye

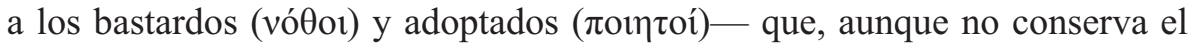
nombre del arconte para la datación exacta, sí contiene el del proponente: Teozótides, bastante raro como para que se pueda pensar que tiene que ser el Cicineo, confirmando - y complementando- el testimonio del cliente de Lisias; en la inscripción se recogen varios nombres de ciudadanos y se alude al escrutinio de los huérfanos y al magistrado que lo verifica ${ }^{4}$. Entre los testimonios acerca de los intentos de limitación de la ciudadanía, destaca el discurso de Lisias XXXIV (Sobre no derrocar la constitución tradicional en Atenas), que se refiere a las propuestas de Formisio. Los intentos de regularización de la ciudadanía se revelan igualmente en Isócrates VIII 88 (Sobre la paz con los lacedemonios); dentro de una argumentación contraria al imperio, para mantener la pureza, quiere evitar que la ciudad se llene de ciudadanos procedentes de fuera. Lisias XXIII (Contra Pancleón) se pronuncia contra alguien que pretendía ser plateense, pero era meteco por haber sido esclavo; el demandante había creído que era meteco, aunque ni siquiera era libre; tenían por ello derecho a apropiárselo. De este modo se revela la relación entre los problemas de la ciudadanía y la evolución de las formas de dependencia tras la guerra del Peloponeso. Demóstenes LVII 18 (Contra Eubúlides) argumenta que Euxiteo ha sido excluido en una revisión por acusaciones en el demo. Habían acusado al padre por su acento, porque lo habían vendido, y a su madre por haber sido nodriza. En general se producen muchos casos judiciales para determinar la falsedad de la ciudadanía a lo largo del siglo IV. Es una consecuencia de largo alcance de la ruptura de la unidad representada por el ciudadano, el granjero y el soldado 5 . En la democracia radical y en la guerra, la identidad del ciudadano se ha alterado con respecto a la ortodoxia de la ciudad hoplítica. Al modificarse las condiciones como consecuencia del

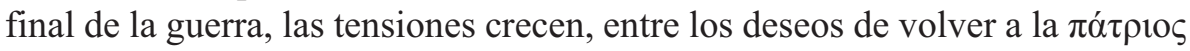
$\pi \mathrm{o} \lambda \imath \tau \varepsilon i ́ \alpha$ por parte de unos y los de conservar los derechos adquiridos en la edad de oro de la democracia por parte de otros.

${ }^{4}$ La inscripción fue publicada por R. Stroud, «Theozotides and the Athenian Orphans», Hesperia 40, 1971, pp. 280-301, que la data en 403/402, en el marco de las distintas regulaciones restrictivas de la ciudadanía.

${ }^{5}$ L. Foxhall, «Farming and Fighting in Ancient Greece», en J. Rich y G. Shipley (eds.), War and Society in the Greek World, Londres-Nueva York, 1993, pp. 134-145. 
Las alteraciones afectan igualmente al otro lado de la sociedad, el de la población dependiente. Ahora hay esclavos con fortuna, como parece ser el caso del Glaucón citado por Esquines I 62 (Contra Timarco), fenómeno

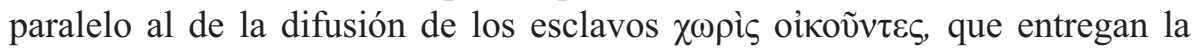
ả $\pi$ фора́ (ibíd. 97) ${ }^{6}$. Los inicios del siglo IV ofrecen el germen de una nueva situación, que sirve de marco a las posiciones individuales que protagonizan el período.

La ciudadanía representa, pues, un elemento clave para comprender las transformaciones habidas en Atenas en los inicios del siglo IV. Las tensiones del momento se revelan en torno a los intentos de establecer restricciones al derecho de ciudadanía bajo diferentes criterios. Por el contrario, Trasibulo de Estiria, que había permanecido incólume en el asunto de las Arginusas, propone en 403 «la ciudadanía a todos los que habían venido con él desde el Pireo», de los que algunos eran claramente esclavos, según Aristóteles, Constitución de los atenienses 40.2; pero se rechaza, y además se recupera la ley

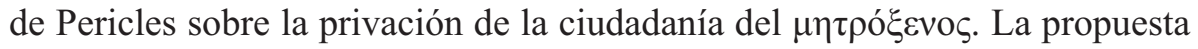
despertó la oposición de Arquino, que también promovió la condena a muerte sin juicio previo de alguien que quería tomar represalias (Isócrates XVIII 2-3 [Recurso contra Calímaco], además de Aristóteles, ibíd.). El Estagirita considera que, al paralizar el decreto de Trasibulo, Arquino contribuyó a la democracia ${ }^{7}$. La salvación de la democracia consistía en establecer limitaciones que hicieran posible su continuidad, aunque hubiera que limitar la ciudadanía y ceder a las presiones restrictivas, para aceptar el «golpe blando» contra los derechos del $\delta \tilde{\eta} \mu o s$, con la amenaza del «golpe duro» de los Treinta ${ }^{8}$. En gran medida se trataba de promover el fin de la democracia, pues la falta de ciudadanía significa la esclavización del pobre, según se desprende de Aris-

${ }^{6}$ Sobre sus privilegios, D. IV 36 (Primera Filipica); XXXIV 6, 37 (Contra Formión); Hyp. III 6 (Contra Atenógenes); E. Perotti, «Esclaves chorìs oikoûntes», en Actes du colloque 1972 sur l'esclavage, París, 1974, pp. 47-56. Sobre banqueros de procedencia servil: Isoc. XVII 12 (Sobre un asunto bancario); D. XXXVI 14, 28-29 (Excepción en favor de Formión) nos hablan de Pasión, manumitido antes de 394, de Formión, su esclavo y hombre de confianza en los setenta, y del matrimonio de la viuda del banquero con el esclavo manumitido.

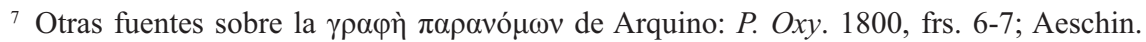
III 195 (Contra Ctesifonte) — cuyo escolio, lo mismo que Plu., Mor. 835F-836A, limitan únicamente a Lisias la fallida propuesta de ciudadanía por parte de Trasibulo- .

${ }^{8}$ D. Plácido, «La formation de dépendances à l'intérieur de la polis après la Guerre du Peloponnèse», Index 20, 1992, p. 148. 
tóteles (Política II $12.5=1274^{\mathrm{a}}$ ), pues para él, si el pueblo no tiene derecho de control y elección de las magistraturas, sería sólo esclavo. Aristóteles (Política III 8.7-8 = 1279³9-1280a 6 ) sabe que oligarquía no es sólo de pocos sino de ricos; los ricos son pocos pero todos comparten la libertad, por lo que unos y otros aspiran a la $\pi$ o $\lambda \imath \tau \varepsilon i ́ \alpha$. De ahí la importancia del debate sobre la ciudadanía al final de la guerra, pues significa la libertad del pueblo o no. La exclusión de esclavos vendría confirmada por Aristófanes, Pluto 1146, donde se ve que Carión, que estuvo en File, sigue siendo esclavo9. Arquino también consigue retener en Atenas a muchos de los ricos oligarcas, a pesar de su participación en los Treinta ${ }^{10}$. El mismo Trasibulo sometió a la Asamblea una nueva moción, ésta sí aprobada, conservada fragmentariamente en piedra y datada en 401/400, bajo el arcontado de Jenéneto, que concedía el privilegio

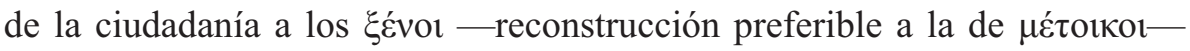
que habían venido de File y a sus descendientes, quedando encuadrados en las diez tribus, así como en tritias y demos, mientras que «aquellos que se habían unido para combatir en la batalla de Muniquia» y «aquellos que habían

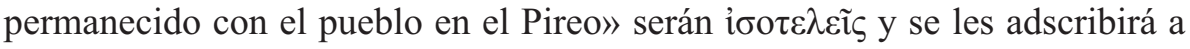
las tribus en virtud del servicio en armas, pero no a tritias ni a demos, con lo que son identificados por sus ocupaciones. Un cálculo del espacio al efecto en los fragmentos de estela revela que la naturalización sólo alcanzó a entre setenta y noventa extranjeros, al núcleo de los que marcharon con Trasibulo a File, posiblemente de estatus hoplítico, en tanto que el resto, entre ochocientos cincuenta y ochocientos setenta, de condición mucho más humilde a juzgar por las faenas en las que se emplean $-\mathrm{y}$ algunos sin duda de origen esclavo, como reprocha Aristóteles-, consiguen la igualdad de cargas con los ciudadanos ${ }^{11}$. Respondería a la promesa hecha en 403, según Jenofonte,

${ }^{9}$ F. Sartori, «Elementi storici del tardo teatro aristofanico e documentazione contemporanea», en Akten des VI. Internationalen Kongresses für Griechische und Lateinische Epigraphik (München 1972), Múnich, 1973, p. 332.

${ }^{10}$ M. Dillon, «Topicality in Aristophanes' "Ploutos"», ClAnt 6.2, 1987, p. 160.

${ }^{11} I G \mathrm{II}^{2} 10$ y 2403; seguimos la lectura de M. J. Osborne, Naturalization in Athens I, Bruselas, 1981, D6, pp. 37-41, y II, Bruselas, 1982, pp. 26-43, y de P. J. Rhodes y R. Osborne, Greek Historical Inscriptions 404-323 B.C., Oxford, 2003, n. ${ }^{\circ} 4$, pp. 20-27, que modifican la de M. N. Tod, A Selection of Greek Historical Inscriptions, II. From 403 to 323 B.C., Oxford, 1948 , n. ${ }^{\circ} 100$, pp. 8-13, no sólo en cuanto a reconocer tres categorías de honorandos, en lugar de dos (aunque la segunda y tercera reciban prácticamente idénticos privilegios), sino también en atribuir la propuesta a Arquino en lugar de a Trasibulo, identificación que había sido seguida mayoritariamente hasta entonces (v. gr. T. Alfieri Tonini, «Sulla proposta di Trasibulo per la 


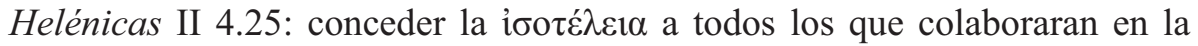
guerra, incluso extranjeros, no sólo metecos ${ }^{12}$. Según Esquines III 187-190 (Contra Ctesifonte), Arquino habría sido por su parte el autor de la propuesta de concesión de una corona de olivo - y no de oro- a cada uno de los «hombres de File» y mil dracmas en su conjunto - menos de diez por cabeza- para sacrificios y ofrendas a la Diosa Madre, ciertamente recompensas mucho más atenuadas que las defendidas por Trasibulo.

A pesar de que el texto de Aristóteles no coincida en la letra con las inscripciones, resulta significativo dentro de su concepción de los límites de la ciudadanía, como cuando le parecía que se alteraban los criterios por el hecho de integrar a los llamados «esclavos metecos» ${ }^{13}$, dado que, desde su punto de vista, la ciudad de los privilegiados estaba delimitada por quienes, por no ser ciudadanos, estaban al borde de la esclavitud.

Los restauradores reciben las alabanzas de Aristóteles (Constitución de los atenienses 40.3) por pagar a los espartanos las deudas de los Treinta y no exigir repartos de tierras. Platón, Carta VII 325B, también reaccionó ante la violencia de la oligarquía y consideraba que se habían evitado las venganzas personales. En la Carta VII $331 \mathrm{E}$ se refiere a las heterías, por las que los propios oligarcas tenían necesidad de protección. En la restauración triunfa la oligarquía moderada. El resultado de la restauración fue el tipo de sistema que elogiará Isócrates en VII 67-69 (Areopagítico), que proclama el dominio sobre otros y la igualdad entre los ciudadanos. Alaba la concordia alcanzada con el restablecimiento del Areópago. También entra en esa línea Demóstenes XX 11-12 (Contra Leptines), que ensalza la política de reconciliación tras la tiranía.

concessione della cittadinanza ateniese», RIL 104, 1970, pp. 154-161, para quien, después de que Arquino parara el intento de Trasibulo de frenar un fortalecimiento de los sectores más humildes de la sociedad, el primero habría propuesto ahora este $\psi \eta ́ \varphi \iota \sigma \mu \alpha$ para evitar asimismo un incremento del poder de los conservadores una vez hubieran regresado los de Eleusis), a pesar de que no encajaba con el perfil político de Arquino y con su actuación de dos años antes. Para D. Whitehead, «A Thousand New Athenians», LCM 9, 1984, pp. 8-10, la inclusión en las tribus revelaría que todos los honorandos recibieron la ciudadanía; por el contrario, P. Krentz, «Foreigners against the Thirty: IG II (2) 10 again», Phoenix 34, 1980, pp. 298-306, y «The Rewards for Thrasyboulos' Supporters», ZPE 62, 1986, pp. 201-204, cree que se les recompen-

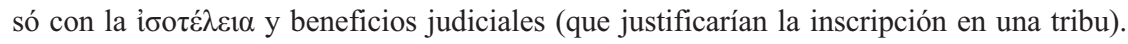

12 J. Hatzfeld, ad loc., CUF, 1936.

13 D. Plácido, «Esclavos metecos», en In memoriam. Agustín Díaz Toledo, Almería, 1985, pp. 297-303. 
Es fácil en estos temas encontrarse con intentos de buscar interpretaciones «facciosas», que traten de encuadrar las distintas actitudes en facciones definidas y relativamente coherentes. Sin embargo, posiblemente sea más fácil tratar de entender las posturas personales en el entramado de intereses del momento, lo que tal vez permita ver aquí un reflejo de la actitud de alguien que, aunque lucha contra los Treinta, tenga reticencias ante las propuestas de concesión de la ciudadanía que podían considerarse excesivas. En definitiva, muchas de las víctimas de los Treinta pretendían sólo favorecer la conservación de la oligarquía sin acudir a los rasgos tiránicos.

A este propósito, es posible volver a considerar la posición de Trasibulo de Estiria, que ha sido oponente de los Cuatrocientos, aunque no ha intervenido en la primera conspiración a que se refiere el discurso de Lisias XIII (Contra Agorato) y, después de 410, se vincularía a Alcibíades y a Terámenes. De hecho, parece haber colaborado al establecimiento de una nueva democracia

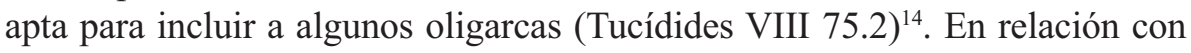
ese momento, Lisias (ibíd. 70-72) se refiere a los beneficiarios del decreto de 410-409 (IG II ${ }^{2} 110=$ GHI 85), donde se incluye Trasibulo Calidonio, premiados por el asesinato de Frínico que provocó el final de los Cuatrocientos. Este Trasibulo recibiría la ciudadanía; en cambio, no hay constancia de la participación de su homólogo de Estiria. Agorato, el objeto del discurso XIII, era un meteco y siguió siéndolo. Habría participado, según él, en el asesinato de Frínico, pero el acusador lo niega. Lisias lo acusaba efectivamente de usurpar la ciudadanía y de haber sido esclavo. El fondo de la acusación, en cualquier caso, era el haber colaborado en los asesinatos de los Treinta.

Formisio, por su parte, propone en cambio reducir la ciudadanía a los propietarios de tierras, pero Arquino impidió su conversión en ley, que habría privado de la ciudadanía a cinco mil atenienses. La propuesta fue rechazada, pero Formisio se relaciona con varios personajes implicados en la restauración, como se ve en Aristóteles, Constitución de los atenienses 34.3.

Lisias compuso en 403 el discurso XXXIV (Sobre no derrocar la constitución tradicional en Atenas) para un político señalado. Formisio era de los

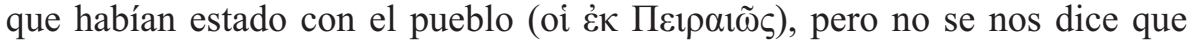
hubiera estado en File. Lo más significativo es precisamente esta actitud de parte de los que han combatido contra los Treinta: han vuelto del exilio para esclavizar la ciudad (§ 2). Para el orador, la salvación de la ciudad depende

${ }^{14}$ W. J. McCoy, «Thrasybulus and his Trierarchies», AJPh 112.3, 1991, p. 315. 
de que todos participen ( $§ 3)$, en lo que sigue la línea de Protágoras, contraria a la propuesta restrictiva de Formisio. El orador compara sus efectos con la oligarquía, porque también la ciudad se privará de caballeros y hoplitas (§ 4), como en aquélla, ya que entonces murieron muchos de los que tienen tierra. La represión no recayó sólo sobre el $\delta \tilde{\eta} \mu o \varsigma$ subhoplítico. En cambio fue precisamente el pueblo el que salvó la ciudad (§ 5). En su opinión, hay que mantener el régimen a pesar de las amenazas de los lacedemonios (§§ 6-7). Daría la impresión de que el orador está refiriéndose a un cambio de régimen hacia la oligarquía, más que a una reducción de la ciudadanía a los que tienen tierra, como se desprende de Dionisio. Para Lisias las restricciones representan el inicio del camino hacia la oligarquía.

Según el mismo orador (§§ 8-10), el imperio había llevado a los atenienses a olvidar su tierra. Con ello, enlaza el problema de la ciudadanía, donde se debatía sobre su restricción o su ampliación, con el económico, provocado por haber dedicado los esfuerzos de la ciudad a la política naval de Pericles y haber desatendido la tierra. Ni siquiera entre los que apoyan medidas democráticas y se oponen a los Treinta e incluso a la reconciliación se recupera el concepto pericleo de Atenas como isla. Será exclusivo del momento más activo del imperio. Tras la guerra, con la falta del imperio, las condiciones de la ciudad y de la democracia se transforman.

Paralelamente a los problemas relacionados con la definición de la ciudadanía y la recuperación del protagonismo de la actividad agraria como objetivo del sistema democrático, se pone de relieve una nueva perspectiva en el uso de los ejércitos mercenarios, que adquieren a partir de ahora un nuevo protagonismo. Uno de los primeros ejemplos del uso de mercenarios de forma masiva fue el del ejército de los Diez Mil, tal como aparece relatado en la Anábasis de Jenofonte ${ }^{15}$. En relación con las preocupaciones territoriales, la construcción de fortificaciones de frontera aparece como una de las preocupaciones de los preparativos de Ciro en Ciropedia III 2.1-24. La Ciropedia parece escrita dentro de un período amplio, tras la guerra de Corinto y antes de la guerra social, en el que Jenofonte aparece como partidario de garantizar algún tipo de dominio exterior, según se desprende del diálogo de Ciro con Tigranes, en III 1.14-31, sobre la base de que demostrar la superioridad crea sumisión voluntaria ${ }^{16}$, sin

${ }^{15}$ L. P. Marinovic, Le mercenariat grec et la crise de la polis, París, 1988, pp. 24-56.

16 J. E. Lendon, «Xenophon and the Alternative to Realist Foreign Policy: Cyropaedia 3.1.14-31», JHS 126, 2006, pp. 82-98. 
necesidad del dominio violento de la época democrática que creaba miedo. El mercenariado adquiere nuevo protagonismo en Jenofonte, Helénicas IV 4.14, donde la guerra parece haber quedado en manos de los mercenarios en 391. Ello requiere la disponibilidad de hombres con necesidad de salario, al tiempo que la proyección de campañas lejanas ${ }^{17}$. De hecho, se creaban nuevos problemas derivados de las necesidades del salario de las tropas, según se desprende de Hel. Oxy. 19(14).2. En ese ambiente se insertan los problemas de Conón con los mercenarios chipriotas de Hel. Oxy. 20(15) ${ }^{18}$. Y por lo mismo, resulta interesante investigar la posible participación de Jenofonte en el suministro de mercenarios peloponesios a Dionisio de Siracusa, lo que se ha relacionado con la redacción del Hierón del año $388^{19}$. Podría proporcionar una clave acerca de la postura de Jenofonte sobre las relaciones entre la ciudad y el poder personal, tal vez extrapolable hacia algunos sectores de la sociedad ateniense, especialmente de los círculos socráticos. La teórica defensa de la sociedad propia de la polis se vería así matizada por la necesidad de una defensa basada en relaciones personales, más que en la solidaridad cívica. El final de la guerra del Peloponeso señala los síntomas de una nueva sociedad, prevista por los Treinta y por sus enemigos.

Los enemigos de los Treinta, cuando se reunieron en el ágora, ya habían solicitado la ayuda de los lacedemonios (Aristóteles, Constitución de los atenienses 38.1). Lisias mismo, XII 55-57 (Contra Eratóstenes), pone de relieve que muchos de los que derrotaron a los Treinta eran simples competidores de éstos, pero agudizaron el enfrentamiento con los del Pireo. Hicieron la guerra a los Treinta y al pueblo, porque los Treinta no ejecutaban a los peores, sino a los que merecían más honores que ellos por linaje, riqueza y virtud. Había oligarcas que culpaban a los Treinta y llamaron a Pausanias, de acuerdo con Lisias, XVIII 10-12 (Sobre la confiscación de los bienes del hermano de Nicias). El discurso está escrito para un hijo de Éucrates, hermano de Nicias, amenazado de confiscación, en un proceso anterior a 395. Éucrates era estratego en el momento del establecimiento de la oligarquía de los Treinta, de los que se negaron a la paz y no quisieron participar en la oligarquía (§ 4), como

${ }^{17}$ Sobre el coste económico de esta guerra de desgaste, C. Fornis, «Campañas espartanas olvidadas: Jenofonte y la fase de desgaste de la guerra de Corinto», Ktèma 32, 2007, pp. 351361.

${ }^{18}$ Véase I. A. F. Bruce, «The Mutiny of Conon's Cypriot Mercenaries», PCPhS 8, 1962, pp. 13-16.

19 A. Giuliani, «Dionigi I, Sparta e la Grecia», RIL 128, 1994, pp. 156-157. 
favorables al sostenimiento de la democracia y tuvieron que beber la cicuta, como se ve en el discurso Contra Agorato 7-8 (XIII), del mismo Lisias. El hijo de Éucrates tiene que luchar por su hacienda y por la ciudadanía (§ 1). Es la historia de algunos oligarcas víctimas de la propia oligarquía, porque ésta pensaba que tenían demasiados honores. Nicérato, el hijo de Nicias, había sido víctima de los Treinta por ser muy inclinado al pueblo (§6), pero, como otros, después de perecer en la oligarquía como favorables al pueblo, en la democracia «somos despojados de los bienes como hostiles al pueblo»

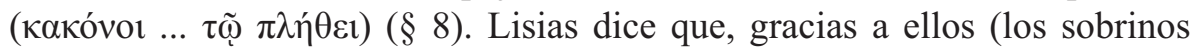

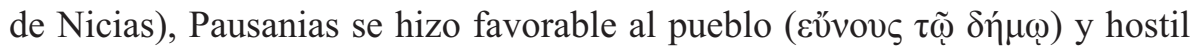
a los Treinta. El otro hermano de Nicias, Diogneto, tuvo que partir al exilio al acabar la oligarquía de los Cuatrocientos (§ 9), aunque no se unió, como otros oligarcas, a los espartanos de Decelia. Éstos fueron los que recibieron el apoyo de Pausanias y los lacedemonios de la Academia (§ 10). El autor concluye que así Pausanias se hizo favorable al $\delta \tilde{\eta} \mu \circ \varsigma(\S 11)$. El orador dice que no se entiende que Pausanias tuviera más piedad que el pueblo ateniense con los oligarcas que al final se hicieron enemigos de los Treinta (§ 12). Considera que evitar las confiscaciones favorece la concordia, el mejor modo de hacer lo que conviene a la multitud (§ 17), para evitar la $\sigma \tau \alpha ́ \sigma ı \varsigma, ~ c a u s a$ de todos los males. La concordia fue el mérito de la restauración (§ 18). El discurso, de 395, se refiere a nuevas amenazas de confiscación, que al autor le parecen menos justificadas que las que se pudieron hacer inmediatamente después de la tiranía (§ 19). Ahora sólo tendría sentido como efecto de la acción de quienes quieren dar muestras de ser leales después de haberse quedado en la ciudad en los momentos clave. Para el orador, lo que hay que hacer es salvar para la ciudad los bienes que ésos quieren confiscar, pues sería provechoso para «vosotros» (los ciudadanos como jueces) tanto como para nosotros, los propietarios $(\S 20)$. Se trata de proteger la propiedad frente a las confiscaciones promovidas por quienes acusan a los que se consideran oligarcas, aunque no parte de los Treinta, los que se apoyaron en Pausanias. Ante el temor de nuevas confiscaciones, los familiares de Nicias acuden a

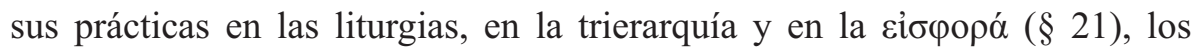
instrumentos con que cuentan los ricos para afirmar su lealtad al pueblo. Han quedado huérfanos bajo los Treinta y ahora están amenazados de perder sus posesiones en la democracia, por el hecho de haber ido a buscar protección para el pueblo junto a Pausanias (\$22). Parece evidente que no todo el pueblo considera favorablemente la intervención de éste, que los protagonistas 
del discurso consideran la salvación. Pide a los jueces que protejan a los que han sido perseguidos por los Treinta (§ 23) por estar a favor de la $\pi$ o $\lambda ı \varepsilon \varepsilon i ́ \alpha$. Sus parientes han muerto en la guerra o víctimas de la cicuta bajo los Treinta en defensa de la democracia y la libertad (§ 24). Los que han sufrido bajo la oligarquía merecen el apoyo de la democracia (§ 25), pues estamos solos por causa de nuestra virtud y de las desgracias de la ciudad. Termina pidiendo que se reconozca a sus parientes como luchadores por la libertad (§27).

Puede considerarse la representación de los oligarcas menos radicales que tuvieron que exiliarse ante el radicalismo de Critias y que volvieron en el mes de septiembre, entre los que están los que Aristóteles (Constitución de los atenienses 34.3), en el momento de la paz tras Egospótamos, considera que no son partidarios de la restauración de la democracia, aunque tampoco per-

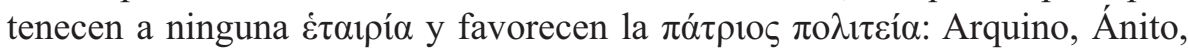
Clitofonte y Formisio, entre los que destacaba Terámenes ${ }^{20}$. Pero el resultado fue la oligarquía de los Treinta con apoyo de Lisandro.

Jenofonte, Helénicas II 4.29, atribuye la iniciativa a Pausanias mismo debido a su rivalidad con Lisandro. Dice (II 4.35) que desunió a los de la ciudad. Promueve la reconciliación con la colaboración de algunos atenienses (4.38), con la promesa de mantener la paz. Trasibulo les reprocha que actúen así siendo ricos (4.40). En 401 hicieron pactos que todavía duran (4.43).

Las tensiones aparecen en Lisias XXVI 10 (Sobre el examen de Evandro), en lo concerniente a la caballería: un aspirante al arcontado (Evandro) es

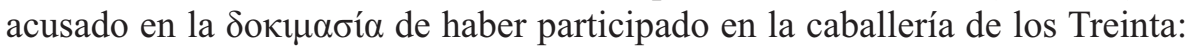
las liturgias en que tanto gastó su padre le sirvieron para destruir la democracia, al haberse ganado la confianza del pueblo ( $(4)$, con implicaciones de Trasibulo de Colito, que era su defensor ( $\$ 21)$; igual que en Jenofonte, Helénicas III 1.4: cuando Tibrón pidió caballeros a los atenienses, éstos los mandaron de los que habían formado parte de la caballería de los Treinta, «en la idea de que sería un beneficio para el pueblo que se marcharan, e incluso que perecieran». Lisias, o el acusador, mantiene activa su actitud contra los Treinta, todavía en el año 382, posible fecha del discurso.

Parece existir en la época una inclinación de los hoplitas ricos a integrarse en la caballería, como se desprende de Lisias XIV 7 (Contra Alcibíades el Joven), donde el sujeto está inscrito como hoplita, pero prefiere servir como caballero:

20 Sobre estos políticos y su filiación ideológica, no siempre fácilmente discernible o etiquetable, vid. supra n. 1. 


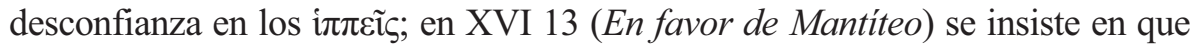
en la caballería no se corría riesgos. Se constata una tendencia a sustituir las funciones de los ejércitos hoplíticos, que se tradujo en la victoria sobre la infantería pesada espartana de 390 (Jenofonte, Helénicas IV 5.3-17, esp. 16) ${ }^{21}$.

Para el acusador de Evandro (§ 5), que ahora haya elegido la tranquilidad no lo libra de los males de la época de la oligarquía. Lo que se usa como prueba contraria a Evandro es la actuación en la oligarquía, no la actuación política en general, como quiere Lateiner ${ }^{22}$. El orador recuerda que la $\delta о \kappa \iota \mu \alpha \sigma i ́ \alpha$ se ha implantado para impedir que desempeñen magistraturas quienes lo han hecho en la oligarquía, en la idea de que no tenía sentido que fueran magistrados de la democracia quienes la habían derrocado (§ 9). Daría la impresión de que se entregan las magistraturas a los oligarcas en vez de los demóticos $(\S 15)$. Hay que distinguir entre los que simplemente permanecieron en la ciudad y los que hicieron daño (§ 16). Evandro era de los que hacían detener y matar (§ 18). Hubo muchos de la ciudad que se sumaron a los exiliados a su vuelta desde el Pireo (§ 19). Son los que el pueblo elige ahora para las

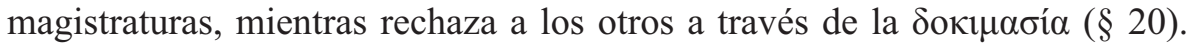
El acusador insiste en que él, en cambio, es más digno de crédito que Trasibulo porque nunca pecó de $\mu 1 \sigma o \delta \eta \mu i ́ \alpha$, ni él ni su padre participaron en la oligarquía (§ 21). El acusador insiste en que sus antepasados se enfrentaron siempre a la tiranía ( $\sigma \tau \alpha \sigma i \alpha ́ \zeta o v \tau \varepsilon \varsigma$ contra ellos) y gastaron mucho en beneficio de la salvación de ciudad ( $\$ 22)$. En el discurso de Lisias se trata de aclarar la compleja situación derivada de los diferentes comportamientos de los miembros de la oligarquía como clase.

El cliente de Lisias XXV (Discurso de defensa por intentos de derrocar la democracia), acusado de conspirar para destruir la democracia, reclama el establecimiento de una diferenciación clara entre los que han colaborado con los Treinta y los que simplemente estaban descontentos con la democracia, sin hacer daño al pueblo ( $§ 11)$. Es una consideración interesante como modo de introducir matices en la comprensión de las actitudes de la posguerra. El mismo orador ( $(9)$ hace constar los cambios de bando de personajes célebres, como Frínico y Pisandro, entre los Cuatrocientos y los

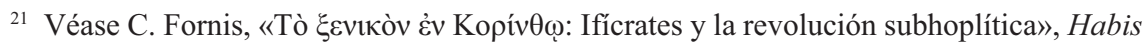
35, 2004, pp. 71-86.

22 D. Lateiner, «"The Man who does not Meddle in Politics": A Topos in Lysias», CW 76.1, 1982, p. 2. 
Treinta. Las diferencias no se basaban en cuestiones políticas, sino en intereses personales $(\S 10)$. El acusado nunca ha tenido problemas con el pueblo y ha desempeñado sus liturgias ( $(12)$. Es injusto atacar a los que no han sido perseguidos bajo la oligarquía, siempre que no hayan sido culpables ante la multitud ( $(18)$. Se puede correr el peligro de caer en los mismos errores (§ 29), actuar como tiranos (§ 30) y castigar a los inocentes sin distinguirlos de los culpables (§ 35).

Como conclusión, puede señalarse que en los conflictos posteriores a la guerra del Peloponeso, tanto a escala individual como colectiva, el protagonismo conceptual corresponde en gran parte a los debates sostenidos en torno a la ciudadanía, en la que se trata de admitir una mayor o menor participación e inclusión, así como confirmar o desechar los derechos de los miembros de la comunidad al margen de su estatuto socioeconómico. En ello se revela la proyección que la ciudadanía tiene como factor protector de las libertades de los atenienses. 